\title{
Echocardiographic method for the estimation of pulmonary artery pressure in chronic lung disease
}

\author{
M J BOYD, I P WILLIAMS, C W G TURTON, N BROOKS, G LEECH, AND \\ F J C MILLARD
}

From the Cardiac Department, St George's Hospital, and Thoracic Medicine Department, St James' Hospital, London

ABSTRACT The prognostic implications of pulmonary hypertension in chronic lung disease, and the difficulty in establishing its severity without cardiac catheterisation, indicate the need for $\$$ reliable non-invasive method of assessing the pulmonary artery pressure. It is likely that the time्ष taken by the right ventricle to generate a sufficiently high pressure to open the pulmonary valve्ठ will increase progressively as the pulmonary arterial diastolic pressure rises. Therefore, the time interval between closure of the tricuspid valve and opening of the pulmonary valve has been $\vec{b}$ obtained from high-speed echocardiographic recordings of the tricuspid and pulmonary valve in a group of 17 patients with chronic lung disease. Each patient underwent right heart cathछ eterisation immediately after the echocardiographic examination so that the pulmonary arteria重 diastolic pressure could be obtained directly. A range for the group from $15 \mathrm{mmHg}(2 \cdot 0 \mathrm{kPa}) \mathrm{t} \stackrel{\vec{\Phi}}{\mathrm{m}}$ $45 \mathrm{mmHg}(6.0 \mathrm{kPa})$ was observed. The linear correlation between the measured diastolic press

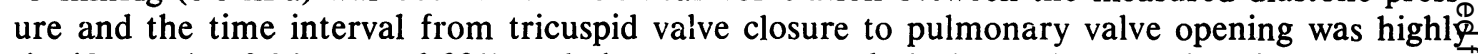
significant $(r=0.94, p=<0.001)$ and the scatter was relatively small. It is therefore suggeste $\vec{\Phi}$ that this time interval, obtained non-invasively by echocardiography, can be used as an index of the severity of pulmonary hypertension associated with chronic lung disease.

Chronic lung disease (CLD) may be complicated by pulmonary hypertension (PHT). This results in right ventricular hypertrophy and is an important factor in the pathogenesis of right ventricular failure when the prognosis is usually poor. ${ }^{1} \mathrm{How}-$ ever, the development of significant PHT is by no means inevitable, and so a means of estimating the pulmonary arterial pressure (PAP) is important for prognostic assessment and may alter subsequent management. At present, detection of PHT, except by direct measurement of PAP at cardiac catheterisation, is unreliable until the process is advanced. The classical auscultatory features of raised PAP are particularly difficult to evaluate in the presence of hyperinflation, as are the radiographic signs, and although some reliance can be placed on the electrocardiogram, ${ }^{2}$ this is too insensitive for any attempt at quantifying the severity of PHT.

Address for reprint requests: Dr MJ Boyd, Department Medicine 1, St George's Hospital Medical School, Cranmer Terrace, London SW17.
There is thus a need for a more reliable non: invasive technique for the evaluation of PHT in these patients. In this study we have explored the possibility that, in the presence of PHT, opening of the pulmonary valve will be delayed, because a greater pressure must be generated by the right ventricle before ejection can occur. The techniqued used has been to make M-mode echocardiographie. recordings of tricuspid and pulmonary valve motion in a group of patients who also underwent right heart catheterisation, and to investigate whether the interval between tricuspid valvew closure and pulmonary valve opening (TVC-PV\& interval) can be used to predict the severity of PHT.

\section{Methods}

Seventeen patients were studied. All but one were men and the age range was 48 to 75 years (table)? All patients had evidence of severe chronic airways? disease with a history of cough or breathlessnes 
Table Summary of results of echocardiographic and catheterisation examinations

\begin{tabular}{|c|c|c|c|c|c|c|c|c|}
\hline \multirow[t]{2}{*}{ Patient } & \multirow{2}{*}{$\begin{array}{c}\text { Age } \\
(y r)\end{array}$} & \multirow[t]{2}{*}{$\operatorname{Sex}$} & \multicolumn{2}{|c|}{ Echocardiogram } & \multicolumn{4}{|c|}{ Catheterisation } \\
\hline & & & $\begin{array}{l}T V C-P V O \\
(m s)\end{array}$ & $\begin{array}{l}\text { Heart Rate } \\
\left(\min ^{-1}\right)\end{array}$ & $\begin{array}{l}P A D P \\
(m m \mathrm{Hg})\end{array}$ & $\begin{array}{l}\text { Mean PAOP } \\
(\mathrm{mm} \mathrm{Hg})\end{array}$ & $\begin{array}{l}\text { Merr RAP } \\
(m m \mathrm{Hg})\end{array}$ & $\begin{array}{l}\text { Heart rate } \\
\left(\text { min }^{-1}\right)\end{array}$ \\
\hline 1 & 48 & $\mathbf{M}$ & 128 & 78 & 45 & 11 & 9 & 83 \\
\hline 2 & 56 & $\mathbf{M}$ & 57 & 81 & 16 & 8 & 4 & 84 \\
\hline 3 & 56 & $\mathbf{M}$ & 38 & 69 & 16 & 8 & 6 & 73 \\
\hline 4 & 57 & $\mathbf{M}$ & 69 & 73 & 21 & 10 & 7 & 79 \\
\hline 5 & 60 & $\mathbf{M}$ & 49 & 88 & 16 & 7 & 3 & 82 \\
\hline 6 & 60 & $\mathbf{M}$ & 86 & 74 & 27 & 7 & 5 & 70 \\
\hline 7 & 61 & $\mathbf{M}$ & 58 & 62 & 20 & 15 & 10 & 66 \\
\hline 8 & 63 & $\mathbf{M}$ & 67 & 78 & 29 & 12 & 12 & 74 \\
\hline 9 & 66 & $\mathbf{M}$ & 52 & 82 & 22 & 7 & 6 & 80 \\
\hline 10 & 67 & $\mathbf{M}$ & 57 & 78 & 22 & - & 11 & 74 \\
\hline 11 & 68 & $\mathbf{M}$ & 116 & 84 & 44 & - & 0 & 82 \\
\hline 12 & 69 & $\mathbf{M}$ & 73 & 69 & 26 & 17 & 10 & 71 \\
\hline 13 & 69 & $\mathbf{M}$ & 61 & 80 (AF) & 22 & 12 & 8 & 88 (AF) \\
\hline 14 & 70 & $\mathbf{M}$ & 60 & 73 & 17 & 11 & 7 & 69 \\
\hline 15 & 72 & $\mathbf{M}$ & 60 & 77 & 25 & 5 & 1 & 80 \\
\hline 16 & 75 & $\mathrm{~F}$ & 65 & 82 & 26 & 18 & 12 & 78 \\
\hline 17 & 75 & $\mathbf{M}$ & 32 & 96 & 15 & 9 & 6 & 95 \\
\hline Mean \pm SD & - & - & - & $77 \cdot 5 \pm 8 \cdot 4$ & - & - & - & $78 \cdot 6 \pm 7 \cdot 2$ \\
\hline
\end{tabular}

$1 \mathrm{~mm} \mathrm{Hg}=0 \cdot 133 \mathrm{kPa}$

Abbreviations-see text

or both for more than three years and a forced expiratory volume in one second less than $50 \%$ of the predicted normal. Two patients (5 and 16) had severe old tuberculous lung fibrosis also, and one (9) had additional cryptogenic fibrosing alveolitis. In order that a wide range of values for PAP might be obtained, no selection criteria based on clinical, electrocardiographic, or radiological suspicion of PHT were applied. Subjects were not included if they had clinical evidence of right ventricular failure with a raised jugular venous pressure, or if the electrocardiogram showed complete right bundle branch block (RBBB pattern with QRS complex duration exceeding $0 \cdot 11 \mathrm{~s}$ ). Informed consent was obtained from each patient before inclusion in the study, which had been approved previously by the ethical committee of St George's Hospital.

Each patient was examined first by echocardiography, and immediately afterwards by right heart catheterisation.

The echocardiographic examination was made with the patient lying supine with approximately $30^{\circ}$ elevation of the head and trunk. Recordings were made using a commercial ultrasound generator (Smith Kline Instruments Ekoline 20) in which the pulse repetition rate had been increased to $2000 \mathrm{~s}^{-1}$, and a focused transducer with a nominal frequency of $2.25 \mathrm{mHz}$. The returning echoes were recorded on photographic paper using a Cambridge six-channel recorder modified to have a paper speed of $200 \mathrm{~mm} \mathrm{~s}^{-1}$.
The normal precordial site for placement of the ultrasound transducer rarely provided satisfactory visualisation of intracardiac structures and in most patients the best recordings were made from the subxiphoid area. From this approach the mitral and aortic valves were identified by the conventional criteria. The tricuspid valve was usually found by directing the transducer towards the aortic valve when the tricuspid valve was seen between the transducer and the aortic valve; slight adjustment of the transducer more posteriorly and to the right was then necessary to reveal the closure point of two cusps of the valve. In order to locate the pulmonary valve, the transducer was directed towards the aortic valve again, and then readjusted upwards and to the left, so that the ultrasound path was dorsal to and almost parallel with the anterior chest wall. (An important practical point to note is that, because the pulmonary artery is at a distance equal to or greater than the aorta from the sub-xiphoid site, the pulmonary valve appears on the echocardiogram recording at a depth similar to or greater than the aortic valve. ${ }^{3}$ This contrasts with the usual precordial transducer site pattern in which the more anterior pulmonary valve is nearer than the aortic valve to the transducer.)

Recordings were made of the tricuspid valve and of the pulmonary valve at $200 \mathrm{~mm} \mathrm{~s}^{-1}$ paper speed, together with simultaneous recording of the standard electrocardiographic lead showing the clearest onset of the QRS complex (fig 1). 


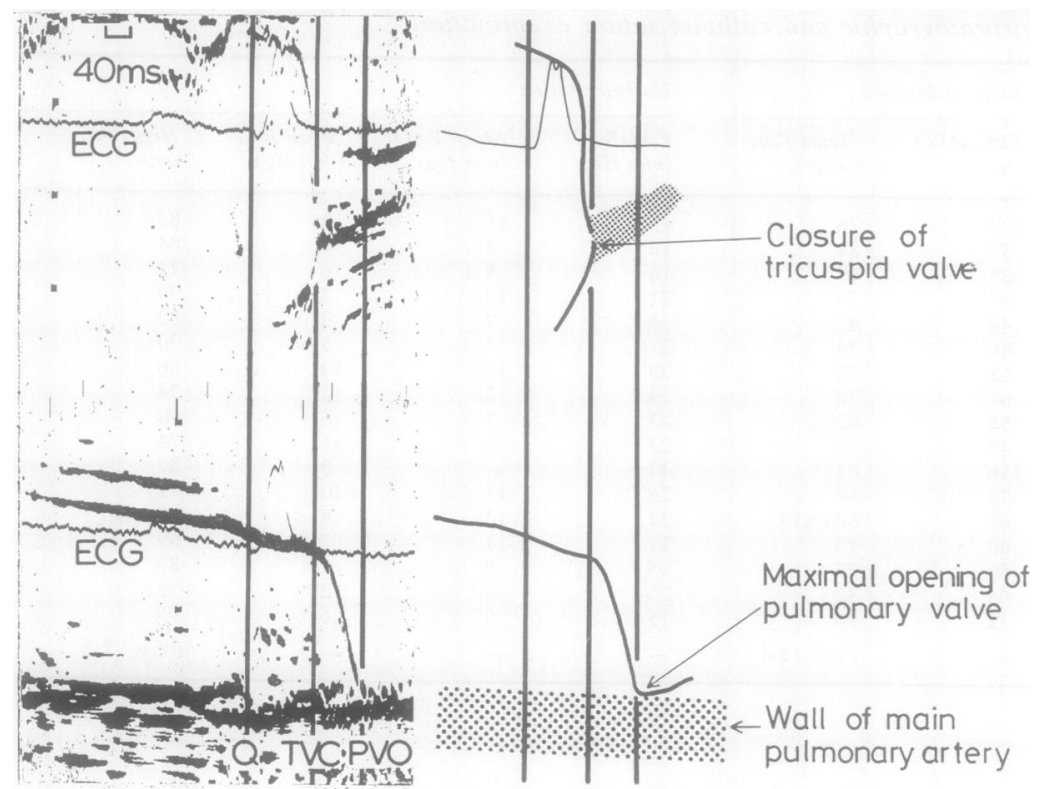

Fig 1 Composite figure showing the echocardiographic appearances of the tricuspid valve (upper half of figure) and the pulmonary valve (lower half of figure) at fast paper speed $\left(200 \mathrm{~mm} \mathrm{~s}^{-1}\right)$ from one of the patients studied (12). The electrocardiograms (ECG) recorded simultaneously with each valve echocardiogram have been aligned vertically along the $Q$ waves (line $Q$ ) of the $Q R S$ complexes in order to illustrate the time relationship of the motion of the two valves. The diagram on the right of the figure indicates the points used for time interval measurement. The tricuspid valve is represented by echoes from the anterior cusp (upper) and septal cusp (lower) which meet at the point of closure of the tricuspid valve as indicated (line TVC). The pulmonary valve is represented by a single echo from one of the cusps, which exhibits a rapid downward deflection when the valve opens. The point of maximal opening is indicated (line PVO), where this downward motion ceases as the cusp meets the wall of the main pulmonary artery. (The lower ECG tracing is omitted from the diagram for clarity.)

Conventional right heart catheterisation was performed with the subject again positioned supine with $30^{\circ}$ elevation of the head and trunk. All pressure measurements were made with reference to the fourth intercostal space, at the midanteroposterior point, from recordings taken during held end-tidal expiration. A no 7 SwanGanz (flow-directed) catheter was introduced into the right median basilic vein under local anaesthesia, and in all patients was advanced satisfactorily to a branch of the pulmonary artery under fluoroscopic control. Recordings were made of mean and phasic right atrial pressure (RAP), right ventricular pressure, and mean and phasic PAP. In all but two patients a pressure measurement could be made during temporary balloon inflation to occlude a branch of the pulmonary arterial tree and thus obtain an indirect estimate (PAOP) of left atrial pressure.

The echocardiographic recordings were analysed by an observer who had no knowledge of the catheterisation data. The average time intervalo from the onset of the electrocardiograph QRS complex to the point of complete closure of theo tricuspid valve (Q-TVC) was calculated from at 3 least five cardiac cycles (10 cycles in the case ofo one patient who had atrial fibrillation). The average time interval from the onset of the $\mathrm{QRS}$. complex to the point of maximal opening of then pulmonary valve (Q-PVO) was similarly obtained. It was then possible to obtain the time interval TVC-PVO by the following subtraction:

TVC-PVO (ms) = Q-PVO (ms) minus Q-TVC $(\mathrm{ms})$ O From analysis of the catheterisation recordings, the value of PADP was derived from the average of five cardiac cycles, and the values for the mean? RAP and mean PAOP were obtained directly.

The heart rate during each procedure was ob $\frac{\text { Dे }}{\mathbb{D}}$ tained by averaging $10 \mathbf{R}-\mathbf{R}$ intervals from the? simultaneous electrocardiograms recorded with the echocardiogram and the catheterisation pressure tracings. 


\section{Results}

The relevant data obtained from both echocardiographic and catheterisation examinations are given in the table. In five patients the pulmonary arterial diastolic pressure (PADP) was at the upper end of the normal range $(16 \mathrm{mmHg}-2 \cdot 1 \mathrm{kPa})^{4}$ and in the other 12 patients the PADP was elevated to a varying degree with the highest recorded value being $45 \mathrm{mmHg}(6.0 \mathrm{kPa})$. Most patients had a normal mean RAP $(\leqslant 8 \mathrm{mmHg}-1 \cdot 1 \mathrm{kPa})^{4}$ but in five there was modest elevation of this up to $12 \mathrm{mmHg}(1.6 \mathrm{kPa})$. However, none had a grossly elevated mean RAP, indicating that clinical exclusion of right ventricular failure before entry to the study had been adequate. Two of the patients who had increased mean RAP also had slightly elevated PAOP $(>15 \mathrm{mmHg}-2 \cdot 0 \mathrm{kPa}),{ }^{4}$ but in neither at a level sufficient to be the cause of PHT. All other patients had a normal PAOP. Only minor

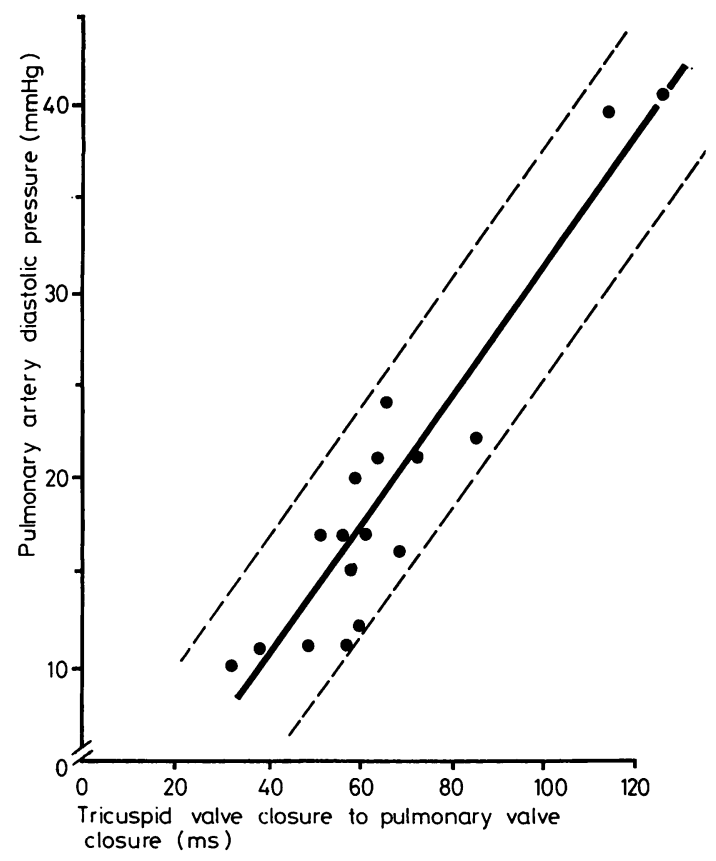

Fig 2 Graph showing the relationship between pulmonary artery diastolic pressure measured at cardiac catheterisation (ordinate) and the time interval from tricuspid valve closure to pulmonary valve opening derived by echocardiograph (abscissa), for which the linear regression equation $(y / x)$ is: $P A D P(m m H g)=0.34(T V C-P V O$ interval in $m s)$ $+1 \cdot 6$. Solid line $=$ line of regression; broken lines $=$ limits of twice the standard error of estimate (SEy) on either side of regression line; $r=0.94 ; p=0.001$; $S E y=3.0 \mathrm{mmHg} .(1 \mathrm{mmHg}=0.133 \mathrm{kPa}$. $)$ changes in heart rate in no consistent direction were observed when echocardiographic and catheterisation data were compared for each patient, and the mean heart rate for the whole group during echocardiographic examination did not differ significantly from that during catheterisation.

The echocardiographic measurement of the TVC-PVO interval ranged from $32 \mathrm{~ms}$ to $128 \mathrm{~ms}$, and fig 2 shows that over the range of measured PADP in this group a highly significant correlation exists between this time interval and the directly measured PADP.

\section{Discussion}

The possibility that echocardiography may be used to assess PHT has aroused interest in recent years. The M-mode technique is particularly suitable for following the rapid movements of heart valves during the cardiac cycle, and several workers have reported characteristic abnormalities of motion of the echo from the pulmonary valve in PHT. Some of these can only be considered qualitative indicators of PHT. They include: reduction in, or absence of, the normal negative deflection that follows right atrial contraction ${ }^{5}$; loss of the normal down sloping direction of the cusp echo during diastole ${ }^{5}$; coarse flutter of the cusp echo during systole (often resulting in a W shaped pattern ${ }^{6}$ ). This last abnormality is not, however, specific for PHT.

The most important parameters reported to bear a quantitative association with PAP are: increasing steepness of the slope of the cusp echo during the opening motion of the valve ${ }^{5}$; and increase in the ratio of right ventricular pre-ejection period to right ventricular ejection time (RVPEP/RVET ratio). ${ }^{7}$ Neither technique appears to have a close enough correlation with PAP to be clinically useful for estimating the degree of PHT. Further, the first of these methods presents a specific problem when used to interpret echocardiograms from the majority of patients with CLD in whom the subxiphoid transducer site must be used to obtain satisfactory recordings. This different anatomical approach may introduce an important error into the index because it is based on measurements made from the depth axis. The second technique ${ }^{7}$ obviates this problem because only time axis measurements are needed, but has the limitation that it is rare to be able to record pulmonary valve closure (and thus obtain RVET) in older subjects, especially if there is hyperinflation, thus restricting this method to paediatric practice. Moreover, RVPEP includes the time interval from 
the onset of the electrocardiogram QRS complex to the onset of right ventricular activation. Cardiac catheterisation studies have indicated that this period has a range of variation of approximately $35 \mathrm{~ms}$ in normal subjects. ${ }^{8}$ This variation is not dependent on PAP and may thus partly account for the rather wide scatter found by these workers ${ }^{7}$ when RVPEP/RVET ratio was related to PAP.

It thus seems that an index based only on time measurement, but which is less affected by factors which are independent of the PAP, might be a more reliable non-invasive way to assess PHT. Leatham and Vogelpoel observed that pulmonary ejection sounds associated with pulmonary valve stenosis (and thus with a low PAP) are almost coincident with the first heart sound, whereas ejection sounds occurring in patients with PHT appear appreciably later in systole. ${ }^{9}$ This phenomenon reflects the longer time period required for the right ventricle, after it has closed the tricuspid valve at the onset of systole, to generate the higher pressure required to open the pulmonary valve when the PAP is raised. Unfortunately, the absence of ejection sounds in many patients with PHT and difficulties in defining the sound of tricuspid valve closure within the first heart sound complex prevent the clinical application of this observation to most patients.

The accuracy with which M-mode echocardiography can time valve motion events ${ }^{10}$ has been used in this study to obtain the TVC-PVO interval as described above. By recording at a fast paper speed, measurements can be made to an accuracy of $\pm 2.5 \mathrm{~ms}$. Small inter-cycle variations of valve timing were found, however (pooled standard deviations for all subjects were $\pm 4.4 \mathrm{~ms}$ for TVC and $\pm 6.8 \mathrm{~ms}$ for PVO), and it is important to average the time measurements from several cycles. Although it would be theoretically preferable to define PVO as the point at which the pulmonary valve begins to open, in practice this point cannot be determined accurately. We have, therefore, taken measurements from the point of maximal pulmonary valve opening. This introduces an additional (but relatively constant) factor into the TVC-PVO interval, but the interval is far more reproducible when measured this way because maximal opening of the pulmonary valve is a well-defined point (fig 1).

In the analysis of the relationship between the TVC-PVO interval and the measured PAP, only the association with PADP has been considered. This is because it is the diastolic component that determines the pressure that the right ventricle must develop to open the pulmonary valve. An association between the TVC-PVO interval and $\frac{\text { ? }}{\circ}$ mean PAP and systolic PAP can be demonstrated $\frac{\bar{\sigma}}{\bar{n}}$ $(r=0.74$ and $r=0.72$ respectively), but this only $\overparen{D}$ reflects a tendency for the systolic and diastolic values of the PAP to rise proportionately in PHT. ${ }^{\infty}$

Minor variations in PADP for a particular indi- $\vec{\circ}$ vidual resulting from changes in heart rate are a $\overrightarrow{\vec{\omega}}$ potential source of error in this study because the $\stackrel{\omega}{\omega}$ echocardiographic recordings and the catheter- $\vec{x}$ isation recordings were not simultaneous. However, no patient showed more than a small change in rate between the two procedures and there was? no consistent pattern in the direction of change. $\stackrel{\theta}{A}$ Moreover, as a whole, the group showed no significant difference in mean heart rate during the two parts of the study. Therefore, we do not $\vec{\nabla}$ believe that these small differences within the ${ }^{\mathbb{D}}$ physiological range have introduced any important $\frac{\Phi}{3}$ error into our results. As can be seen in fig 2 응 the association between TVC-PVO interval and PADP is highly significant and it is suggested that $\vec{\oplus}$ this method provides an estimate of the true PADP: for the heart rate during the examination.

Care has been taken to exclude from the study any patient in whom other factors are present which might affect right heart valve timings. Firstly, a high RAP will result in the tricuspid $\mathbb{D}$ valve closing much later in right ventricular $\overrightarrow{\vec{F}}$ systole when the right ventricular pressure has $\frac{3}{3}$ already increased considerably. This causes a shortening of the TVC-PVO interval and so this: technique cannot be applied to patients who have overt right ventricular failure with a raised jugularo venous pressure. Secondly, an increase in the $\underset{x}{\stackrel{0}{\nexists}}$ TVC-PVO interval has been demonstrated ino some patients who have complete right bundle branch block, ${ }^{11}$ and so no patient with this electro-o̊ cardiographic abnormality has been included in the study.

One further limitation of this technique that re-D quires comment is the technical difficulty of recording echocardiograms in patients with CLD and hyperinflation. The pulmonary valve is the most difficult valve to record and is only able to be found in $75 \%$ to $80 \%$ of normal subjects. As ex- $\omega$ pected the success rate is less when there is hyper inflation, but in our experience, the associatedo flattening of the diaphragm facilitates echocardio- $\mathbb{\Phi}$ graphic recordings of good technical quality from ${ }^{\text {? }}$ all the heart valves in approximately $60 \%$ of patients with CLD when the transducer is directed $\frac{0}{\mathbb{D}}$ from the subxiphoid area.

In conclusion, our results indicate that thee TVC-PVO interval can be obtained non-invasively by an echocardiographic technique in patientso 
with CLD, and that the linear correlation between this interval and the directly measured PADP shows sufficiently little scatter for this interval to be of value clinically in the estimation of the severity of PHT in these patients. It is likely to be particularly useful for serial examinations of the same patient, and further work is needed to study its use in this way.

\section{References}

1 Ude AC, Howard P. Controlled oxygen therapy and pulmonary heart failure. Thorax 1971; 26: 572-8.

2 Millard FJC. The electrocardiogram in chronic lung disease. Br Heart $J$ 1967; 29:43-50.

3 Chang S, Feigenbaum H, Dillon J. Subxiphoid echocardiography. Chest 1975; 68:233-5.

4 Hurst JW (ed). The heart: arteries and veins. Third edition, 1974:83. New York: McGraw-Hill.

5 Nanda NC, Gramiak R, Robinson TI, Shah PM.
Echocardiographic evaluation of pulmonary hypertension. Circulation 1974; 50:575-81.

6 Weyman AE, Dillon JC, Feigenbaum H, Chang S. Echocardiographic patterns of pulmonic valve motion with pulmonary hypertension. Circulation 1974; 50:905-10.

7 Hirschfeld S, Meyer R, Schwartz DC, Korfhagen J, Kaplan S. The echocardiographic assessment pulmonary artery pressure and pulmonary vascular resistance. Circulation 1975; 52:642-50.

8 Braunwald E, Fishman AP, Cournand A. Time relationship of dynamic events in the cardiac chambers, pulmonary artery and aorta in man. Circ Res 1956; 4:100-7.

9 Leatham A, Vogelpoel L. The early systolic sound in dilation of the pulmonary artery. $\mathrm{Br}$ Heart $J$ 1954; 16:21-3.

10 Mills, P, Craige E. Echophonocardiography. Prog Cardiovasc Dis 1978; 20:337-58.

11 Brooks N, Leech G, Leatham A. Complete right bundle-branch block: echophonocardiographic study of first heart sound and right ventricular contraction times. Br Heart J 1979; 41:637-46.

\section{The Dr H M (Bill) Foreman Memorial Fund}

The Trustees of the above fund invite applications for fellowships from medical practitioners in the study of respiratory disease. Grants for travel to, and support for clinical research in, countries other than the applicant's own are available. Intending applicants should write for further details to Dr B H Davies, Sully Hospital, Sully, South Glamorgan, UK. 